\title{
A study of the status of the elderly; bacterial infections, their causes, prevention, and control methods
}

\begin{abstract}
Assar Sh, $\mathrm{MSc}^{1}$, Mirzaei T, $\mathrm{PhD}^{2,3^{*}}$, Ravari A, $\mathrm{PhD}^{2,3}$, Vakilian $\mathrm{AR}, \mathrm{PhD}^{4,5}$, Rezahosseini $\mathrm{O}$, $\mathrm{MD}^{6}$, Hosseini F, $\mathrm{MSc}^{7}$, Assar S, DDS, $\mathrm{MS}^{8}$

1- Instructor of Medical Bacteriology, Geriatric Care Research Center, Rafsanjan University of Medical Sciences, Rafsanjan, Iran. 2- Assistant Prof, Geriatric Care Research Center, Rafsanjan University of Medical Sciences, Rafsanjan, Iran. 3- Assistant Prof, Dept. of Medical Surgical Nursing, Rafsanjan University of Medical Sciences, Rafsanjan, Iran. 4- Associate Prof, Geriatric Care Research Center, Rafsanjan University of Medical Sciences, Rafsanjan, Iran. 5- Associate Prof. of Neurology, Dept. of Neurology, School of Medicine, Rafsanjan University of Medical Sciences, Rafsanjan, Iran. 6- Resident of Infectious Diseases, Dept. of Infectious and Tropical Diseases, Tehran University of Medical Sciences, Tehran, Iran. 7- MSc of Nursery, Geriatric Care Research Center, Rafsanjan University of Medical Sciences, Rafsanjan, Iran. 8- Assistant Prof, School of Dentistry, Hormozgan University of Medical Sciences, Bandar Abbas, Iran.
\end{abstract}

Abstract

Received: October 2016, Accepted: January 2017

Background: On the $26^{\text {th }}$ anniversary of the International Day of Older Persons in 2015 with the slogan "the elderly, advocates of dynamism, growth, and the youth of the population", 12\% (900 million individuals) of the world population were above 65 years of age. It is estimated that this rate will reach $22 \%$ ( 2 milliard individuals) by 2050 . The purpose of the present study was to determine the status of the elderly and bacterial infections, their causes, prevention, and control methods.

Materials and Methods: In the present study, Magiran, SID, CAB Direct, PubMed, CDC, and World Health Organization (WHO) databases were searched using the keywords bacterial infections, old age, and control methods. The inclusion criteria included articles related to the study topic in English or Farsi. After the assessment of the articles, 40 articles were studied in detail.

Results: Due to the relative increase in the duration of life and diseases related to old age, the recurrence of some infections has been observed in the elderly. Immune system dysfunction, lifestyle, living area, elderly care method, and underlying diseases can be effective on aging and acquiring of infectious diseases. In elderly patients, infections, unlike in young adults, do not have evident symptoms. More than $30 \%$ of cases of mortality among the elderly are due to infectious diseases. The most common infections in the elderly are urinary tract, respiratory, unspecified, intra-abdominal, skin, and catheter-related infections.

Conclusions: Infectious diseases are of grave importance in the quantity of life and quality of life (QOL) of the elderly. Thus, the determination of their causes, the most common infectious diseases, and their causal factors in the world, developing countries, and Iran can play an important role in the management of these diseases.

Keywords: Elderly, Infection, Mortality, Iran

\section{Introduction}

Until now, the World Health Organization (WHO) has not reached an accurate overall and universal number for the onset of old age. In developed countries, this age has been considered as 65 and older. In other areas, including Africa, in order to receive services provided for the elderly, those older than 60 are

\footnotetext{
* Corresponding author: Tayebeh Mirzaei, Geriatric Care Research Center, Rafsanjan University of Medical Sciences, Rafsanjan, Iran.

E-mail: t.mirzaei@rums.ac.ir
} 
considered as elderly, and the WHO has also recognized this age as the onset of old age (1). Physiological aging is the decline in functional capacity due to excess tissue loss compared to tissue growth and production during youth (2). In terms of sociology, the elderly, based on the year and era they were born in, have experienced different cultural, social, and economic circumstances compared to their previous generations. It is necessary that needs and expectations of each generation be considered and met by the related establishments. In 1900, $1 \%$ (15 million individuals) of the world population was above 65 years of age; however, in 2000 , this rate was about $10 \%$ (606 million individuals). On the $26^{\text {th }}$ anniversary (2016) of the International Day of Older Persons with the slogan "Take a Stand Against Ageism"

(3), 12\% (900 million individuals) of the world population were above 65 years of age. It is estimated that this rate will reach $22 \%$ (2 milliard individuals) by 2050 (3-5). According to the last national census in Iran in 2011, the Iranian elderly constitute $8.2 \%$ of the population and it has been predicted that our country will face an explosive increase in elderly population by $2031(6,7)$.

There is little evidence of the better health status of the elderly compared to their parents. The most common disruption in the health status of the elderly are non-communicable diseases (NCDs) such as cardiovascular diseases (CVDs), cerebrovascular ischemia, chronic pulmonary diseases, back and neck pain, chronic obstructive pulmonary disease, depressive disorders, falls, diabetes, dementia, and osteoarthritis (OA) (5). The health of the elderly is not related to biological age; some elderly individuals have the physical and mental ability of 20-year-old individuals. In some countries, including the USA, individuals of 65-84 years of age can be observed who are in perfect health and can perform all their daily activities independently. Aging in good health is achievable and is a process that enables individuals to perform the activities they wish to $(5,6,8)$.

Today, ageism occurs more often than racism or sexism and can have serious consequences for the elderly and society. Discriminatory attitudes and practices and the prevention of the elderly from voicing their point of view will negatively impact the quantity and quality of their health and social care services. Hence, the performance of societies must change in order to create a positive attitude toward aging and the health of the elderly and to observe their human dignity. The cost of social and health cares for the elderly may be considered as a burden; however, it is more appropriate if these costs are considered as a real investment and encourage the elderly to perform the great work they have in mind (5). Nevertheless, in some cases, the elderly are subject to financial abuse or negligence (9). The famous sentence “... when an old man dies, it's a library burning." was written by a writer from Mali named Amadou Hampate $\mathrm{Ba}$ in 1962 (10). Therefore, the aging of the population must not be considered as a problem even in developing countries. The increasing elderly population illustrates the possibility of preservation and transference of knowledge and competence to younger generations. Thus, providing care for the elderly and meeting their needs in developing countries must not be considered as charity work, but as a necessity for the survival and development of countries $(11,12)$.

In the $21^{\text {st }}$ century, all countries must have an integrated system for long-term care for the elderly; however, some countries have made no preparations in this regard and must start from scratch. Some others must review their longterm care policies and extend their support for vulnerable individuals to the establishment of dignity and independence for all elderly individuals. It has been predicted that by 2050 the number of elderly individuals who will need 
this support will be 4 times the present number. Moreover, in order to ensure healthy aging, all governmental departments and sectors must work toward this goal and have programs and policies for the provision of prepared housing, housing construction, and appropriate transportation services. Furthermore, work environments must make the presence of all age groups possible. Poverty among the elderly must be prevented through community protection schemes. These require further optimization of the status of the elderly and greater understanding of issues related to age and its increasing trend (7). Previous findings suggest that CVDs, motor vehicle accidents, cancer, and intentional and unintentional injuries are the major causes of death in the Iranian population. Many years of life are lost due to motor vehicle accidents, CVDs, cancer, and intentional and unintentional injuries. No comprehensive data was found on the prevalence of infectious causes of death in Iranian elderly individuals in previous studies; however, the prevalence of the causes of death among the elderly is provided in figure 1 (13).

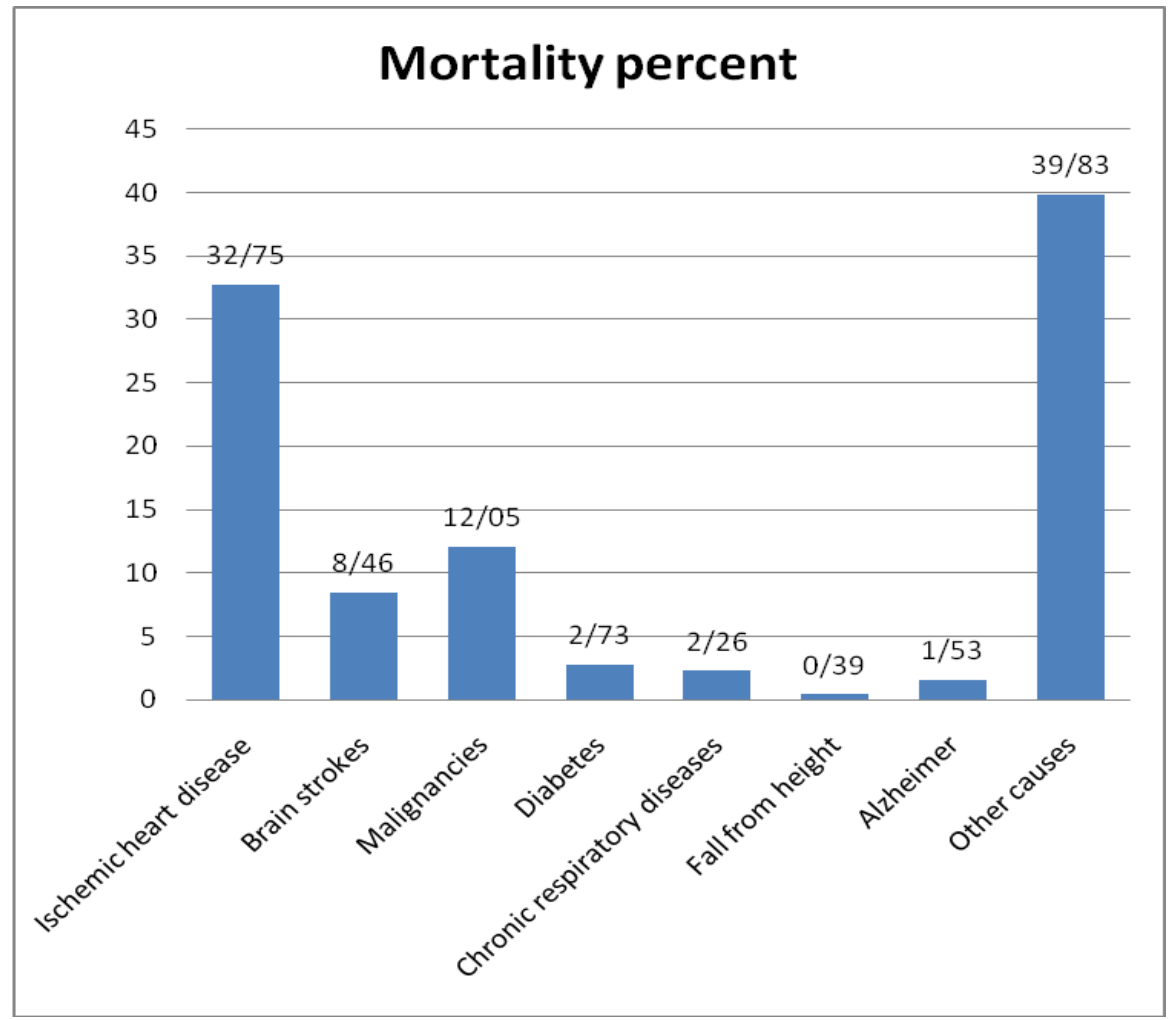

Figure 1: Prevalence of the causes of death among the Iranian elderly

\section{Material and Methods}

This review study was conducted to study the status of the elderly and bacterial infections, their causes, prevention, and control methods. Related articles were found by searching Magiran, SID, CAB Direct, PubMed, CDC, and WHO databases using the keywords bacterial infections, old age, and control methods. Some articles were found using the references of other articles. The study criteria included articles related to the study topic in English or Farsi and the exclusion criteria included one-page articles and letters to the editor. After the assessment of the articles, 40 articles were studied in detail. 
Each article was evaluated in terms of the studied concepts. Moreover, the researchers attempted to answer the following questions: what is the status of bacterial infections in old age? What are the causes of bacterial infections in old age? How can infections be treated in the elderly? How can bacterial infections be prevented in the elderly?

The final stage of this review was the organization and categorization of the obtained content which is presented in the results section.

\section{Results}

The main themes obtained consisted of infectious diseases and the elderly, the context for the emergence of infections in the elderly, most common bacterial infections in the elderly, treatment of bacterial infections in the elderly, method of prevention of bacterial infections in the elderly, the status of bacterial infections during old age in developing countries, and status of bacterial infections during old age in Iran.

Infectious diseases and the elderly: Since the dawn of civilization until today, infectious diseases have had a central role in the quantity and quality of life (QOL). Infectious diseases were considered as the main cause of mortality throughout the world until the mid- $20^{\text {th }}$ century. Life expectancy was short due to illnesses such as the plague, typhus, cholera, typhoid, diphtheria, pneumonia, tetanus, and severe wound infections. Complications due to infections with some bacterial diseases such as syphilis, leprosy, smallpox, measles, scarlet fever, rheumatic fever, pertussis, and poliomyelitis would threaten human life. After the recognition of the relationship between microbes and infectious disease and the discovery of causative agents of many of these diseases, methods of pollution removal from animate and inanimate surfaces, disinfectants, vaccines, and antibiotics, the rate of mortality due to microbial agents was reduced and life expectancy increased. However, other diseases such as atherosclerosis, cardiovascular disease (CVD), cancer, diabetes mellitus (DM), chronic diseases of the joints, and osteoporosis were reported as the common causes of mortality. Based on the fact sheet S310 of the WHO updated on May 2014, the 10 main causes of death during 2000-2012 were, respectively, ischemic heart disease (IHD), stroke, lower respiratory tract infection (LRTI), chronic obstructive pulmonary disease (COPD), lung cancer, AIDS, DM, road accidents, and hypertension $(14,15)$.

Presently, due to the relative increase in lifespan and illnesses related to old age, we are faced with the re-emergence of some infections that can have a considerable effect on the quantity of life and QOL of the elderly. The most common reason for the referral of the elderly to emergency units and their hospitalization is infectious disease $(16,17)$. Thus, a large part of the treatment costs of individuals, which is usually related to old age, is due to frequent visits to the doctor, duration of hospitalization, medication costs, and hospital costs. Furthermore, $30 \%$ of cases of mortality among the elderly are due to infectious diseases (18). This is due to the fact that infectious diseases in the elderly, compared to the youth, have no clear symptoms and even family members and caregivers may not recognize that the changes in the elderly are related to infectious diseases (19, 20).

Context for the emergence of infections in the elderly: Immune system dysfunction, lifestyle, living area, and life program can be effective on aging, and thus, its resulting issues $(8,21,22)$.

Immune system deficiency: Regarding immune system deficiency, we can refer to its dysfunction and power, fundamental changes in compliant immunity, chronic diseases, medication use, malnutrition, and deficiency in body systems. $\mathrm{T}$ lymphocytes are subject to variation in terms of production and 
reproduction and their interaction with macrophages (IL-2 production) decreases. In addition, antibody production against new antigens is reduced. Moreover, the drying and thinning of the skin and mucous membranes, the enlargement of the prostate and its resulting complications, reduced cough reflex, and thus, the swallowing of pharyngeal secretions, or any issues in the anatomical and physiological status of the body impact innate immunity and can increase susceptibility to infection. Chronic diseases such as cancer, atherosclerosis, DM, kidney failure, arthritis, and dementia (alone or in combination) can result in immune system deficiency and increased susceptibility to some infections. Analgesics, narcotic drugs, cholinergic drugs, and antacids can reduce the performance of innate immunity and render the elderly more susceptible to infections. Reduced acid and intestinal peristalsis in the elderly can facilitate gastrointestinal infections (23). On the other hand, 30-60 percent of the elderly do not have a suitable diet which also weakens the immune system. Research has shown that the impact of nutrition on the weakening of the immune system is greater than was previously imagined. Moreover, $11 \%$ of the elderly are depressed, have DM, and suffer from the side effects of medications, all of which may play a role in their malnutrition. Malnutrition in particular has affected the elderly in developing countries and economic growth has resulted in a rapid change in diet, in the form of increased consumption of fat and animal products and reduced consumption of fresh vegetables. This phenomenon is called the transmission of diet and has led to higher rates of obesity and chronic diseases within the society which, in turn, increases the risk of infection in the elderly because obesity results in chronic diseases such as DM. Stress also affects the immune system efficiency and indirectly facilitates infection acquisition. Poor function of body systems such as lack of motor function, urinary and fecal incontinence, dysphagia, disruption in blood flow, or delayed wound healing or all of these cases in combination can increase susceptibility to infection. This may be the cause of the elderly having the highest rank among patients with nosocomial infection $(8,18,19,24-26)$.

Factors related to lifestyle: Of the factors related to lifestyle which increase susceptibility to infection, the retirement period, desire to travel and visit relatives or different places, and exposure to contaminants can be noted. Volunteering, visiting hospitalized friends, or other social life patterns can increase the risk of infection. Usually, the elderly refer to health and treatment centers more often due to their health issues; therefore, they are at greater risk of contagious diseases due to the high population at these centers. The elderly prefer ready-made food which can increase the risk of foodborne infection such as Campylobacter, Clostridium perfringens, Escherichia coli O157:H7, Salmonella, and Staphylococcus aureus. Residence in nursing homes, whether daily or permanently, is another factor which can increase their risk of infection. Thus, a healthy lifestyle, regular exercise, a balanced diet, regular medical care, maintenance of personal hygiene, and observation of food preparation and handling are of great importance $(8,19,23$, 27, 28).

Factors related to area of residency: The elderly use either private residency areas or areas managed by trained and experienced nurses. There are also apartments or single rooms that can be provided to one individual or an elderly couple. Moreover, there are residency areas which provide food, sleeping areas, showers, transportation, and assistance in dressing and areas for the elderly without any family members and elderly individuals who are unrelated, but have similar care needs. There is no accurate data on residency area and the difference between the mentioned nursing homes in terms of susceptibility to infection, but 
there is a higher rate of nursing homes-related infections (8). At these locations, the most common infections are related to the urinary tract, the lower respiratory tract, especially the lungs, and the skin and soft tissue. In the USA, for every 1000 days of residency in nursing homes, 3-7 cases of infection, or 6.1-8.3 million cases of elderly infections in proportion to the population, have been reported $(8,19,27,28)$.

Low prognosis and suspicion of infection in the elderly: In 2012, 33-40\% of cases of mortality among those above 65 years of age were due to infectious diseases. These diseases can also be the underlying cause of mortality due to other illnesses. Diagnosis of infection in early stages is difficult in the elderly due to the lack of its common symptoms such as fever and increased white blood cells. The manifestation of infection is different in the elderly and the common symptoms of infection are not observed in them even in the most severe cases $(29,30)$. Clinical manifestations of disease in the elderly are nonspecific, and for accurate diagnosis and management of disease in the elderly, awareness of the diversity of symptoms in this age group is necessary $(19,29)$.

In 30-50 percent of elderly individuals with reduced body size and infection, fever is not observed; however, a fever of $38.3{ }^{\circ} \mathrm{C}$ can be a sign of infection in the elderly (18). In elderly patients, bloodstream infections (bacteremia), unlike in young adults, are not accompanied with fever, chills, and sweating that can play a role in the lack of diagnosis of infectious diseases in their early stages. The presence of even low-grade fever in the elderly can have grave importance, because in $90 \%$ of case in this age group, the cause of illness is infection. Furthermore, considering that infection in one body system may not have the typical symptoms, it is necessary to pay attention to and assess all body organs of the individual $(18,30)$. The risk of an infection and death due to it in the elderly is several times higher than that of the same infection in young individuals. This may be due to underlying diseases, long-term residency in nursing homes, use of tubes and invasive devices such as intravenous or urinary catheters, gastric feeding tube, and tracheostomy, and reduced physiological reserves. Diagnostic sampling, which requires the cooperation of the patient, such as sputum, pleural fluid, and cerebrospinal fluid (CSF) sampling, is difficult especially in individuals with reduced body size and weakness (31).

In the USA that has a more coherent statistical system, bacterial pneumonia (90\% of cases in individuals of older than 65 years of age) and influenza account for one-fourth of the causes of death in the elderly. Urinary tract infection, which can be in the form of urea bacterium without symptoms, intra-abdominal sepsis, and bloodstream infections caused by gram-negative bacteria, constitutes 5-35 percent of deaths in young individuals, but 37-50 percent of deaths in elderly individuals. Infective endocarditis, tuberculosis, skin and soft tissue infections, septic arthritis, and herpes zoster are other common infections in the elderly. Among the mentioned diseases, pneumonia and urinary tract infection are the most common in the elderly. Of the possible signs of infection in the elderly, increased confusion, changes in mental status and functional status, such as lack of sufficient urinary control and inability to perform activities they were capable of until only a few days back, issues in walking, falling (which may be natural and due to reduced body size and BMI and result in injuries in the elderly), gasping, changes in appetite, and worsening of health status can be noted (30).

The most common bacterial infections in the elderly: The most common infections in the elderly, respectively, consist of urinary tract infections (25-55 percent), respiratory infection (10-34 percent), unspecified infection (11-31 percent), intra-abdominal infection (9-20 percent), and skin and catheter-related infections 
(9\%) (17, 19). Other common infections are herpes zoster (shingles) and fungal nail infection (32). Among the urinary tract infections, E. coli, Proteus mirabilis, Klebsiella, Enterobacter, and coagulase-negative staphylococci are, respectively, the most common. In addition, if the patient has a urinary catheter, the risk of multifactorial infections (microbial) will be increased. The rate of mortality due to urinary tract infection is 5-10 times higher among the elderly compared to young individuals. It is noteworthy that, based on the colony count (bacteria) per unit volume of urine of these individuals compared to young individuals (100 thousand bacteria per $\mathrm{ml}$ of urine), infection is possible with much fewer bacteria in the urinary tract of these individuals. Therefore, the consideration of the patient's age in the interpretation of test results is important (33). Since $11 \%$ of urinary tract infection cases in the elderly have no symptoms, urine culture is a necessary routine test (34).

The second most common infection in the elderly is respiratory infection in the form of pneumonia. Its resulting mortality rate in the elderly is higher than (3 times) other age groups; it results in death in $90 \%$ of cases in the elderly. Streptococcus pneumoniae and influenza virus are the most common factors in pneumonia, are the third leading cause of mortality in the world, and account for $7 \%$ of 56 million cases (3-4 million individuals per year) of death per year. Pneumonia is the cause of $90 \%$ (3-5 times that in young individuals) of deaths in individuals of older than 65 years of age. This rate will most probably increase in individuals with poor oral hygiene (tooth decay, gum disease, different microbial flora due to denture use, and etcetera) or underlying diseases (35). Moreover, in longterm care facilities for the elderly, the rate of pneumonia due to Staphylococcus aureus, uncommon bacteria, and gram-negative rodshaped bacteria, such as Haemophilus influenzae, Pseudomonas aeruginosa, and
Legionella pneumophila, is increasing and 30\% of the elderly in these facilities are infected. The most important causative factors of bacterial pneumonia in the elderly are, respectively, Streptococcus pneumoniae, E. coli, Klebsiella pneumonia, Pseudomonas aeruginosa, Haemophilus influenzae, Legionella pneumophila, and Staphylococcus aureus. Annual use of influenza and pneumococcal vaccines can decrease the mortality rate in these individuals by $20 \%$. Thus, prevention in the elderly can include immunization, cessation or reduction of smoking, and lack of use of invasive treatment methods for accompanying diseases in order to minimize the risk of aspiration after myocardial infarction (17). In this case, the use of consciousness-lowering drugs and sedative drugs is a better option (17). The third most common infection in the elderly is intra-abdominal infection, in which abdominal pain and rigidity is not evident. The most important causative factors of this infection are, respectively, Clostridium difficile and enteric gram-negative bacilli (Enterobacteriaceae) (36).

Skin infections can be caused by Methicillinresistant Staphylococcus aureus (MRSA) acquired from the community and gram-negative bacteria. However, their most common causative factors are Staphylococci and Streptococci (both gram-positive bacteria) which, in addition to their severe and aggressive form, may be found in the form of necrotizing fasciitis. Vancomycinresistant enterococci (VRE) are another causative factor of skin infections in this age group (32). Factors which are more common in this age group are cellulitis especially in the lower calves, Erysipelas, necrotizing fasciitis, folliculitis, impetigo, and boil (32, 36). Malignant otitis externa (swimmer's ear) usually occurs during old age and its most common causative factor is Pseudomonas aeruginosa (37). Infections in joints and other artificial parts occur due to gram-positive cocci such as Staphylococcus aureus and coagulase-negative 
staphylococci. In some cases, these infections are polymicrobial infections $(24,37)$.

Bacterial infection treatment in the elderly: Antibiotic treatment is not very efficient in the elderly because its distribution, metabolism, excretion, antibiotic interaction, and side effects are different in the elderly. In the elderly, even in the absence of disease, kidney function follows a decreasing trend. Increased resistance of bacteria toward antibiotics such as MRSA or VRE is another issue of infection treatment during the elderly period. Therefore, it is recommended that an infectious diseases specialist be consulted in the treatment of infections in the elderly, so that after the assessment of the mentioned items, considering the individual's status and the risk of changes in the natural flora, and thus, antibiotic resistance, the appropriate drug is used. It is noteworthy that antibiotics must be immediately administered to the elderly hospitalized in intensive care units (ICUs), but in other cases antibiotics must be used after consultation. The role of the infection consultant can be the determination and use of the appropriate drug, reduced use of antibiotics, prevention of complications caused by antibiotics, and reduced costs. Moreover, many interactions between antibiotics and other medications must be considered in the elderly $(19,37,38)$.

Bacterial infection prevention in the elderly: Since the duration of hospitalization and residency in care facilities such as nursing homes is long for the elderly, compliance with health standards can reduce the spreading of drug-resistant organisms. Hand washing, immunization through influenza and pneumococcal vaccines, use of combination vaccines for adults (diphtheria-tetanus) especially for those living in nursing homes, and use of influenza and hepatitis vaccines in those caring for the elderly can also be effective (12, $20,30,39,40)$.
The status of bacterial infections in the elderly in developing countries: Numerous studies have shown that developing countries are faced with an increased rate of bacterial resistance. These bacteria are related to infections acquired from the community and hospitals and consist of Neisseria meningitides, Salmonella, Streptococcus pneumoniae, Mycobacterium tuberculosis, MRSA, E. coli, and Plasmodium falciparum. This resistance is observed more among the elderly and its rate is increasing. For instance, the Committee for the Prevention and Control of Nosocomial Infections in Argentina has reported Ciprofloxacin in the E. coli bacteria, Proteus mirabilis, and Klebsiella pneumonia in Argentinian outpatients with urinary infection, and that there is higher resistance in patients of older than 65 years of age compared with younger adults. Reduced success in Mycobacterium tuberculosis treatment in the elderly may increase the rate of transmission of drug-resistant tuberculosis. In developing countries, which lack suitable control over the sale and administration of antibiotics, the rate of multidrug-resistant bacteria has increased. This in the absence of some antibiotics can cause an increase in drug-resistant bacteria $(12,39,41)$.

The status of bacterial infections in the elderly in Iran: In a study conducted in the infection ward of Booali Hospital in Qazvin, Iran, during 1 year (2000-2001), from among 240 elderly individuals, $20 \%, 19.5 \%, 13.3 \%$, $4.17 \%, 2.5 \%, 2.08 \%$, and $18.75 \%$ of cases were hospitalized due to pneumonia, urinary and gastroenteritis infection, septicemia, tuberculosis, brucellosis, meningitis, and other infectious (cutaneous and articular infection) and noninfectious causes, respectively (42). In a study in the Golestan Gatroenterology and Hepatology Research Center of $5^{\text {th }}$ Azar Hospital in Gorgan, Iran, of the total of 128 hospitalized elderly individuals, 8 individuals were hospitalized due to unknown causes and died 
due to sepsis (43). However, in the 100 individuals who recovered, the first cause of infection was unknown and the second was pneumonia (43).

Of the 272 individuals hospitalized in the infection ward of Emam Reza Hospital in Mashhad, Iran, in 2007-2008, 37\% were elderly individuals (44). Their most common complaint was respiratory system issues and the most common diagnosis was pneumonia, but the cause of infections could not be determined (44). In 2009, a study was performed in the infection ward of Shahid Beheshti Hospital of Kashan, Iran, on 248 elderly individuals who had been hospitalized during that year (42). The most common complaint of the patient's was fever and dyspnea; however, the most common underlying illness was hypertension and the most common infections were pneumonia and sepsis (42). A study was conducted on 350 elderly patients of over 65 years of age in Valiasr Hospital in Birjand, Iran, in 2009-2010 (45). More than half of the patients had a history of underlying diseases; the most common underlying disease was hypertension. The most common infections were pneumonia and enterocolitis; their prevalence was, respectively, higher in spring, autumn, winter, and summer (45). In the first 6 months of 1997, 101 elderly individuals were hospitalized in the infection ward of Emam Khomeini Hospital in Tehran, Iran (18). The highest rate of febrile infection in these patients were, respectively, related to pneumonia, urinary infection, sepsis, cellulitis, fever with unknown cause, malignancy, meningitis, enterocolitis, brucellosis, and other causes (18). A study was performed on 65 elderly individuals (over 65 years of age) and 65 young individuals (under 65 years of age) in Loghman Hakim and Booali Hospitals of Tehran (31). It was found that the most common possible sources of infection in the elderly group (case group) were pulmonary infection and urinary tract infection, respectively. In the young individuals group (control group), the most common possible sources of infection were, respectively, gastrointestinal infection and pulmonary origin infections (31).

\section{Discussion}

Individuals of over 65 years of age are more at risk of systemic infections due to various reasons. One of the most important reasons is delay in recognition of the infection by the family, caregivers, and the patient. Moreover, elderly individuals are more susceptible to infections due to the presence of underlying illnesses. Equipment used in health care services, such as urinary catheter, feeding tubes, artificial joints, and intravenous ports, are common bacteria inoculation sources. Rapid control of the respiratory tract, control of tissue fluid return (edema), diagnosis and treatment of septic status can relatively decrease infection and death. The treatment of infection in the elderly is more difficult than that in young individuals, and requires the consideration of the basic health of the liver and kidneys when using certain drugs. In individuals who use various medications, drug interactions must also be considered. Infections differ in terms of treatment method, discharge from the emergency ward, hospitalization, antibiotic selection, and potential side effects of antibiotics. It is noteworthy that lack of suitable treatment of simple infections in the elderly can increase the rate of infection and mortality by 5 times. On the other hand, the elderly are more susceptible to health care-related infections and nosocomial infections. With the aging of the world population, the susceptibility of the elderly is also increasing. The involved personnel may have worked in many services and then, the range of infections related to the elderly and the possibility of infectious diseases emergence may increase. Infection management specialists and epidemiologists working in hospitals believe that the health care system must be more attentive to 
the aging of the population and are seeking novel strategies for the prevention of infections related to health care services. They may find ways to prevent the transmission of different infectious diseases to the elderly during hospital admittance. The pattern obtained from the few studies conducted in Iran on elderly infections and their common causes shows that many measures must yet be taken for the assessment of these infections.

\section{Conclusion}

The order of elderly infections in Iran is similar to that of other large countries of the world. Moreover, similar to developing countries, more efforts should be made regarding the provision of comprehensive care for the elderly. Furthermore, various authorities responsible for public health, especially the Ministry of Health, must address the future explosive increase in elderly population; in the past decade the number of students in our country was higher than 20 million individuals and these individuals will soon reach old age.

\section{Acknowledgement}

We would like to thank the Geriatric Care Research Center (GCRC), Rafsanjan University of Medical Sciences, for their kindly supports.

Conflict of interest: None declared.

\section{References}

1. World Health Organization. Health statistics and information systems, Proposed working definition of an older person in Africa for the MDS Project. Geneva, Switzerland: WHO; 2010. Available from:

http://www.who.int/healthinfo/survey/ageingdefn older/en/index.html

2. Sheykhi MT. State of widowhood in Iran: Challenges of ageing spouses. International
Journal on Ageing in Developing Countries 2016; 1(1):54-67.

3. John B, Keeler LW, Wiek A, Lang DJ. How much sustainability substance is in urban visions?-An analysis of visioning projects in urban planning. Cities 2015; 48:86-98.

4. World Health Organization. Ageing and life course, Facts about ageing. Geneva, Switzerland: WHO; 2014. Available from: http://www.who.int/ageing/about/facts/en/

5. World Health Organization. Health topics, Ten facts on ageing and health. Geneva, Switzerland: WHO; 2015. Available from: http://www.who.int/features/factfiles/ageing/en/

6. Noroozian M. The elderly population in Iran: an ever growing concern in the health system. Iran $\mathbf{J}$ Psychiatry Behav Sci 2012; 6(2):1-6.

7. Nemati D, Agha Bakshy H. Tehran is an elderly lover city, primary steps of global of elderly capital city. Social Research 2013; 6(18):15-44.

8. Strausbaugh LJ. Emerging health care-associated infections in the geriatric population. Emerg Infect Dis 2001; 7(2):268-71.

9. Alizadeh-Khoei M, Sharifi F, Hossain SZ, Fakhrzadeh H, Salimi Z. Elder abuse: risk factors of abuse in elderly community-dwelling Iranians. Educ Gerontol 2014; 40(7):543-54.

10. Bridgewater PB. Biosphere reserves: special places for people and nature. Environ Sci Policy 2002; 5(1):9-12.

11. Sabeghi H, Nasiri A, Zarei M, Kazemi Tabar A, Golbaf D. Respecting for human dignity in elders caring in perspective of nurses and elderly patients. Medical Ethics Journal 2015; 9(32):4570.

12. Gavazzi G, Herrmann F, Krause KH. Aging and infectious diseases in the developing world. Clin Infect Dis 2004; 39(1):83-91.

13. Namazi Shabestari A, Saeedi Moghaddam S, Sharifi F, Fadayevatan R, Nabavizadeh F, Delavari A, et al. The most prevalent causes of deaths, DALYs, and geriatric syndromes in Iranian elderly people between 1990 and 2010: findings from the global burden of disease study 2010. Arch Iran Med 2015; 18(8):462-79.

14. Centers for Disease Control and Prevention and The Merck Company Foundation. The State of aging and health in America 2007. Whitehouse Station, NJ, United States: The Merck Company Foundation; 2007. Available from: https://www.cdc.gov/aging/pdf/saha_2007.pdf

15. World Health Organization. Media centre, The top 10 causes of death. Geneva, Switzerland: WHO; 2014. Available from: 
http://who.int/mediacentre/factsheets/fs310/en/in dex4.html

16. Burlaud A, Mathieu D, Falissard B, Trivalle C. Mortality and bloodstream infections in geriatrics units. Arch Gerontol Geriatr 2010; 51(3):e106-9.

17. Caterino JM. Evaluation and management of geriatric infections in the emergency department. Emerg Med Clin North Am 2008; 26(2):319-43.

18. Rasoolinejad M, Haji Abdolbaghi M, Moradi Mazhari M, Majd Shirazi M. Study of the causes of fever in elderly. Tehran University Medicine Journal 1998; 3(56):41-5.

19. High KP. Infection as a cause of age-related morbidity and mortality. Ageing Res Rev 2004; 3(1):1-14

20. Mertz D, Johnstone J, Krueger P, Brazil K, Walter SD, Loeb M. Adherence to hand hygiene and risk factors for poor adherence in 13 Ontario acute care hospitals. Am J Infect Control 2011; 39(8):693-6.

21. Farzianpour $F$, Hosseini $S$, Rostami M, Pordanjani SB, Hosseini SM. Quality of life of the elderly residents. Am J Appl Sci 2012; 9(1):71-4.

22. John J, Miletic K, Kaye KS. Skin and skin structure infections in older adults. Curr Geriatr Rep 2015; 4(1):70-8.

23. Smith JL. Foodborne illness in the elderly. J Food Prot 1998; 61(9):1229-39.

24. Kammerlander C, Braito M, Kates S, Jeske C, Roth T, Blauth M, et al. The epidemiology of sports-related injuries in older adults: a central European epidemiologic study. Aging Clin Exp Res 2012; 24(5):448-54.

25. High KP. Nutritional strategies to boost immunity and prevent infection in elderly individuals. Clin Infect Dis 2001; 33(11):1892-900.

26. Ghebremedhin B. Bacterial infections in the elderly patient: focus on sitafloxacin. Clin Med Insights Ther 2012; 4:185-200.

27. Kale SS, Yende S. Effects of aging on inflammation and hemostasis through the continuum of critical illness. Aging Dis 2011; 2(6):501-11.

28. Rakhshani T, Shojaiezadeh D, Bagheri Lankarani K, Rakhshani F, Kaveh MH, Zare N. The association of health-promoting lifestyle with quality of life among the Iranian elderly. Iran Red Crescent Med J 2014; 16(9):e18404.

29. Limpawattana $P$, Phungoen $P$, Mitsungnern $T$, Laosuangkoon W, Tansangworn N. Atypical presentations of older adults at the emergency department and associated factors. Arch Gerontol Geriatr 2016; 62:97-102.
30. Mouton CP, Bazaldua OV, Pierce B, Espino DV. Common infections in older adults. Am Fam Physician 2001; 63(2):257-68.

31. Aminzadeh Z, Parsa E. Relationship between age and peripheral white blood cell count in patients with sepsis. Int J Prev Med 2011; 2(4):238-42.

32. Scheinfeld $\mathrm{N}$. Infections in the elderly. Dermatology Online Journal 2005; 11(3):8.

33. Rowe TA, Juthani-Mehta M. Urinary tract infection in older adults. Aging health 2013; 9(5): 10.2217/ahe.13.38..

34. Farajzadeh Sheikh A, Jomehzadeh N, Amin M, Asadi Rad A. Prevalence of asymptomatic bacteriuria in elderly referred to outpatient clinics in Talegani hospital, Abadan, Iran. Jundishapur J Microbiol 2011; 4(3):147-51.

35. Terpenning M. Geriatric oral health and pneumonia risk. Clin Infect Dis 2005; 40(12):1807-10.

36. Spangler R, Van Pham T, Khoujah D, Martinez JP. Abdominal emergencies in the geriatric patient. Int J Emerg Med 2014; 7:43.

37. Prendki V, Zeller V, Passeron D, Desplaces N, Mamoudy P, Stirnemann J, et al. Outcome of patients over 80 years of age on prolonged suppressive antibiotic therapy for at least 6 months for prosthetic joint infection. Int $\mathrm{J}$ Infect Dis 2014; 29:184-9.

38. Beckett C, Harbarth S, Huttner B. Special considerations of antibiotic prescription in the geriatric population. Clin Microbiol Infect 2015; 21(1):3-9.

39. Gavazzi G, Krause KH. Ageing and infection. Lancet Infect Dis 2002; 2(11):659-66.

40. Mirzaei T, Ravari A, Mirzaei S, Loripoor M. Standard precautions: compliance to precautions or precautions as a barrier: a qualitative study. Journal of Qualitative Research in Health Sciences 2014; 3(3):230-8.

41. Talebi-Taher M, Moosavi SAJ, Taherian S, Barati M. Surveying the inappropriate drug adminstrution using beers criteria in elderly patients at the internal medicine ward of Rasoule-Akram hospital of Tehran in 2012. Arak Medical University Journal 2014; 17(2):33-9.

42. Assefzadeh M, Ghasemi R, Zoghi F. Common infections of elderly patients admitted in Bou Ali Sina teaching hospital. Journal of Birjand University of Medical Sciences 2005;12(1-2):5360.

43. Golsha R, Golshah E, Jalali Z, Rajabli N, Roshandel Gh. Final diagnosis and outcomes in old patients admitted to the infectious diseases 
ward. Medical Journal of Mashhad University of Medical Sciences 2012; 55(3):158-63.

44. Tavanaei S, Mirkarimi HR. Evaluation of etiology of infectious diseases in elderly patients admitted to the infectious ward of Imam Reza hospital. Medical Journal of Mashhad University of Medical Sciences 2011; 54(1):31-7.
45. Ziaee M, Sharifzadeh GhR, Mirzaei J, Lotfi L. Admission reasons and common clinical findings in elderly patients were admitted in infectious disease wardof Vali-e-Asr hospital in Birjand in 2009-2010. Modern Care Journal 2012; 8(4):20511. 